\author{
Jeff Cerny, Spencer Kee, Farzin \\ Goravanchi, Elizabeth Rebello and \\ Alicia M Kowalski* \\ Department of Anesthesiology \& Peri-Operative \\ Medicine, MD Anderson Cancer Center, Houston \\ Texas, USA \\ Dates: Received: 17 April, 2014; Accepted: 30 July, \\ 2015; Published: 03 August, 2015 \\ *Corresponding author: Alicia M Kowalski, \\ Department of Anesthesiology \& Peri-Operative \\ Medicine, MD Anderson Cancer Center, Houston, \\ Texas, USA, 1515 Holcombe, Box 409, Houst6on, \\ Texas, 77030, Tel: 713-792-6911; Fax: 713-563-5608; \\ E-mail: amkowalsk@mdanderson.org \\ www.peertechz.com
}

\section{Case Report \\ Peri-Operative Takotsubo Cardiomyopathy: A Case Series}

\section{Introduction}

Stressful events are known to be associated with physiologic insults. Takotsubo Cardiomyopathy (TC) is a reversible, stressinduced, non-ischemic cardiomyopathy associated with temporary weakness of the myocardium and midventricular or apical ballooning $[1,2,4]$. Angina, ST abnormalities, elevated troponins, ventricular asynergy, congestive heart failure (CHF), and decreased ejection fraction (EF) are all components of TC. The unique finding is that they occur on the absence of occlusive coronary artery disease (CAD).3 In this case series with institutional review board (IRB) approval we report three cases of post-operative cardiac symptoms that all resulted in a diagnosis of TC.

\section{Case Report}

\section{Pt. \# 1}

72 year old female presents for cystoscopy/ transuretheral resection of bladder tumor/ exam under anesthesia/ ureteroscopy.

Preop 12-lead electrocardiogram: normal sinus rhythm

Pertinent Past Medical History:

Three years prior: The patient was hospitalized for a cardiac event following a cystoscopy. She complained of angina. Her electrocardiogram showed newly inverted T waves in I, II \& ST elevation V1, 2. An echocardiogram was completed that showed an ejection fraction (EF) of $60 \%$, and mild apical wall hypokinesis. She then underwent cardiac catheterization and was found to have non-occlusive coronary artery disease, with an EF $40-45 \%$. At that time, she was diagnosed with Takotsubo cardiomyopathy by her cardiologist.

Patient had an uneventful anesthetic and cystoscopy on this occasion. The Anesthesia team was hyper-vigilant for any TC recurrence, but no incident occurred.

\section{Pt \#2}

57 year old female with a multinodular goiter, presents for total thyroidectomy.

\section{Pertinent Past Medical History:}

\section{Tobacco use and hiatal hernia with controlled GERD}

In the post-anesthesia care unit (PACU), she developed severe chest pressure 9/10. The 12-lead electrocardiogram did not show any ST-T changes. Anti-acids were given to treat any reflux. Subsequently, her pain decreased to $3 / 10$. However, cardiac enzymes were still sent and the troponin came back at 0.67 . A cardiology consult was called. Symptomatic medical management was immediately implemented.

The patient remained stable overnight with sustained chest pressure $2 / 10$.

Repeat 12-lead electrocardiogram was unchanged. The second troponin was 4.1, with the CK-MB 17.9, and CK 304, consistent with acute non-ST elevation myocardial infarction. Despite being post-op from surgery in the airway, anticoagulation was initiated with close monitoring of the surgical site.

Within 24 hours a cardiac catheterization was performed, with no angiographic evidence of coronary artery disease. However, the patient demonstrated akinesis of the left ventricle and a severely reduced ejection fraction of $30 \%$. In the face of the two diagnostic criteria, she was diagnosed with TC. A repeat echocardiogram was performed the following day, and demonstrated recovery of cardiac function with an ejection fraction to $46 \%$. The patient was discharged symptom-free.

At a two week follow-up appointment, another echocardiogram was performed. This showed full recovery of the myocardium with an EF of 55-60\%, 12-lead electrocardiogram showed sinus rhythm at 70, an "essentially normal EKG".

\section{Pt\#3}

63 year old female with ovarian carcinoma presents for a port-acath (PAC) insertion.

Pre-operatice echocardiogram revealed an EF of $60-65 \%$, and normal left ventricular wall motion.

Pertinent Past Medical History: 
Hypertension, hypothyroidism, hyperlipidemia, GERD, cardiomyopathy, migraines, osteoporosis, depression, diverticulosis (perforated colon secondary to a ruptured diverticulum), and metastatic ovarian cancer with peritoneal carcinamatosis.

Further investigation into the medical record was made specifically targeting the cardiomyopathy diagnosis in the medical history, yet in face of normal EF.

Six months prior, the patient underwent exploratory laparotomy, lysis of adhesions, and tumor reduction surgery. During her postoperative recovery period, the patient reported angina. Cardiac workup revealed mildly elevated enzymes, and 12-lead electrocardiogram showed NSR with non-specific $\mathrm{T}$ wave changes, which "may be due to the myocardial ischemia" in the anteroseptal distribution. Echocardiogram showed EF 45\% with moderately reduced left ventricular function, regional wall motion abnormalities, and a minimal pericardial effusion. A heart catheterization was indicated, and arteriogram revealed no occlusive disease. The left ventriculogram showed mid to distal wall hypokinesis, which correlated with the electrocardiogram changes. In light of these physiologic changes and the absence of occlusive coronary disease, the pathognomonic criteria for TC resulted in her diagnosis. Ultimately she was discharged, and subsequent echocardiogram showed a stable EF of $45 \%$, associated with an improvement in functional capacity.

In PACU, following the PAC placement, the patient developed PVC's. Chest x-ray confirmed proper catheter placement. Lab work revealed hypomagnesaemia of 1.7 . The patient was treated, and the PVC's ceased.

\section{Discussion}

In the surgical setting, an acute $\mathrm{MI}$ and $\mathrm{TC}$ are indistinguishable. Only once occlusive CAD is excluded, then TC is considered. TC is a reversible, stress-induced, non-ischemic cardiomyopathy characterized by temporary weakness of the myocardium and midventricular or apical ballooning, without the presence of occlusive atherosclerosis $[1,2,4]$. Angina, ST abnormalities, elevated troponins, ventricular asynergy, $\mathrm{CHF}$, and decreased $\mathrm{EF}$ are all components of TC. The onset of TC is triggered by a stressful or emotional event in about $90 \%$ of patients. 2 Catheterization is required to confirm diagnosis. Pathognomonic for TC is LV apical wall ballooning (not corresponding to any specific coronary artery territory3) in the presence of normal coronary arteries; there is a lack of CAD on angiography [5].

The etiology of TC is unclear, however evidence points to activation of the sympathetic nervous system: patients are found to have high levels of circulating catecholamines, up to 7-34 times the normal value, compared to $2-3$ times the normal level for patients experiencing acute MI $[2,6]$.

One study by Deshmuku, et al. looked at risk factors for TC: 6,837 patients were diagnosed with TC among 33,506,402 hospitalizations in the Nationwide Inpatient Sample database [4]. The reviewers analyzed comorbidities, and found that patients with hyperlipidemia, hypertension, smoking, alcohol abuse, anxiety state, depression, and stress had higher odds of developing TC (Figure 1-with permission).

Additional risk factors for TC include patients experiencing acute

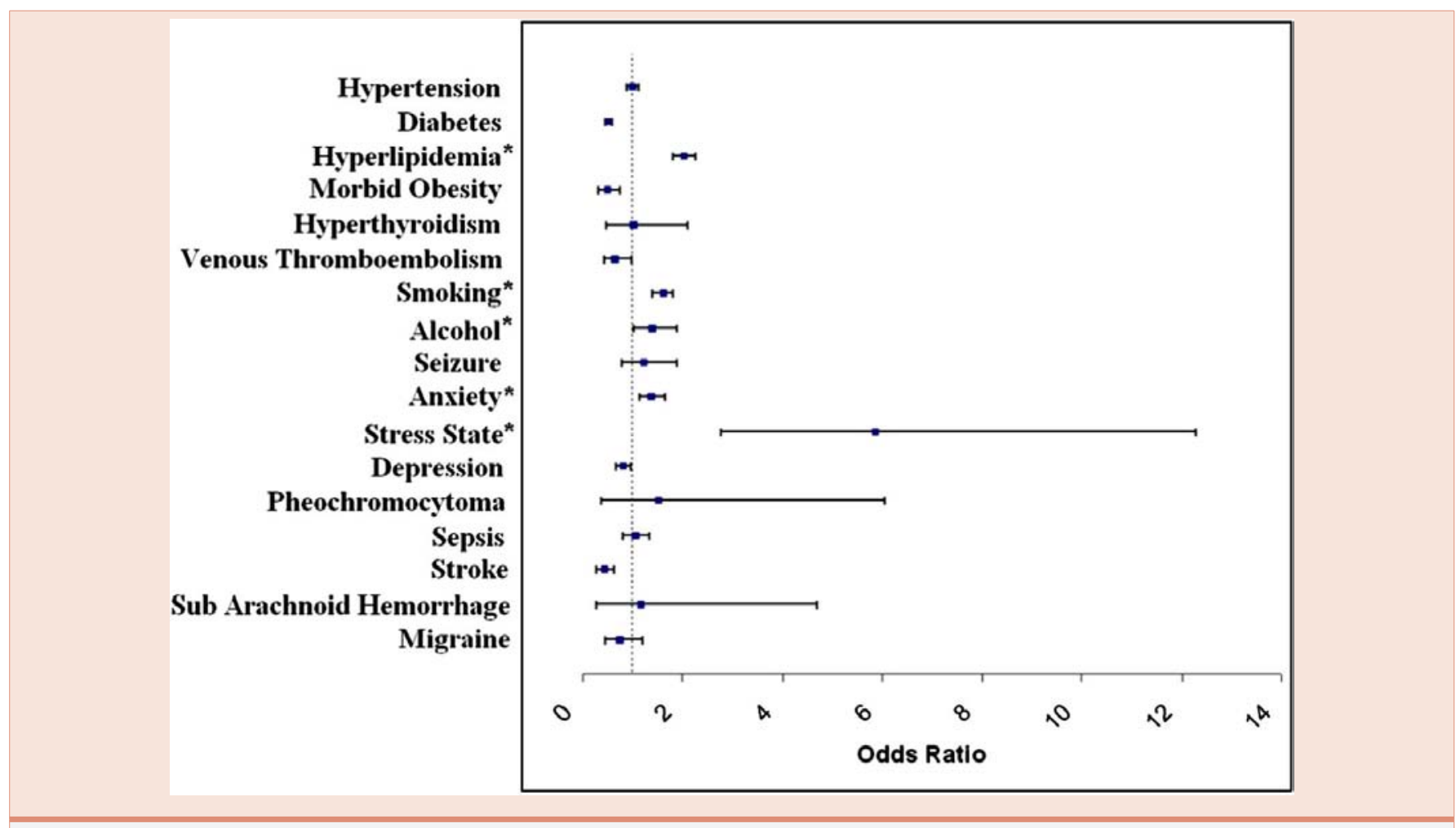

Figure 1: Forest plot showing association of various comorbidities with $\mathrm{TC}^{4}$ (with permission). 
medical illness, acute intracranial events, surgical procedures, or exposure to high levels of endogenous or exogenous catecholamines $[6]$.

Curiously, women are found to have higher odds of developing TC (ratio to men of $>6: 1$ ) [7], and women $>55$ yo 4.8 times higher vs. women $<55$ yo.4 Asian and Caucasian women are thought to have a higher incidence [5]. Contributing factors to this are thought to be smaller left ventricular size, hormonal disturbances, and decreasing vagal tone and baroreflex sensitivity in postmenopausal women [7].

Further, it has been suggested that some women have an exaggerated physiologic response to stress, either through an increased release of or sensitivity to catecholamines, and that postmenopausal women have reduced levels of cardioprotective estrogen [6].

Rarely, complications can occur such as cardiac arrhythmias, congestive heart failure, hypotension, Torsade de Pointes, mitral regurgitation, left-sided heart failure, left ventricular thrombosis, and death (mortality ranging between 1-3\%) [5,8,9]. Fortunately TC carries a favorable prognosis. Treatment is goal-directed towards reducing the myocardial workload and offloading the SVR. Interventions can vary from supportive care with medication management to intraaortic balloon pump in those having acute LV failure [6]. Typically, $\mathrm{LV}$ function is restored within days to weeks, with $33 \%$ of patients having spontaneous recovery of LV function by the time of discharge [6], and $95 \%$ of patients experiencing a full recovery within $4-8$ weeks, and mortality being $<2 \%[5,8]$. Interestingly, the recurrence rate of TC is estimated anywhere from $3-10 \%[1,6,9,10]$. Knowing this rate of recurrence, the anesthesia team in the first case cited above was alert to the possibility of incident peri-operatively. Appropriate concessions, consultations and plans were all made and implemented.

"If patients continue to experience further signs and symptoms or the ejection fraction remains low, another diagnosis should be considered" [6]. This is paramount in the management of a perioperative patient with an acute coronary syndrome. Although the prognosis of TC is favorable, the mortality of a patient with a definitive myocardial ischemic event in the perioperative period carries a mortality rate of $36 \%$ [11]. Thus, it is important to distinguish between the two because the prognosis is so different.

\section{Conclusion}

Takotsubo cardiomyopathy is more common than once thought. In the perioperative setting, signs and symptoms that suggest a myocardial infarction can be due to TC. It is important to have a heightened level of awareness regarding the stress-induced cardiomyopathy, since it occurs in the absence of coronary artery disease and history of cardiac comorbidities. It is also important in patients with a history of TC to remember that it can reoccur in up to $10 \%[1,6,9,10]$. Fortunately, with proper interventions, TC has a favorable prognosis and cardiac function returns to normal.

\section{References}

1. Emanuele Cecchi, Guido Parodi, Cristina Giglioli, Silvia Passantino, Brunella Bandinelli, et al. (2013) Stress-Induced Hyperviscosity in the Pathophysiology of Takotsubo Cardiomyopathy. The Am J Cardiol: 1523-1529.

2. Sharkey SW, Lips DL, Pink VR, Maron BJ (2013) Daughter-Mother TakoTsubo Cardiomyopathy. The Am J Cardiol 137-138.

3. Bielecka-Dabrowa A, Mikhailidis DP, Hannam S, Rysz J, Michalska M, et al. Takotsubo cardiomyopathy -The current state of knowledge. Int J Cardiol:120-125.

4. Deshmukh A, Kumar G, Pant S, Rihal C, Murugiah K, et al. (2012). Prevalence of Takotsubo cardiomyopathy in the United States. Am Heart J. 164: 66-71. e1.

5. Redmond M, Knapp C, Salim M, Shanbhag S, Jaumdally R (2013) Use of vasopressors in Takotsubo cardiomyopathy: a cautionary tale. $\mathrm{Br} \mathrm{J}$ Anaesth 110: $487-488$.

6. Pore N, Burley M (2012) When a broken heart is real: Takotsubo cardiomyopathy. Nurse Pract 37: 48-52.

7. Madias JE (2013) Is the "rare" Takotsubo syndrome a common affliction? Am J Cardiol 111: 778.

8. Kurisu S, Kihara Y (2012) Tako-tsubo cardiomyopathy: clinical presentation and underlying mechanism. J Cardiol 60: 429-437.

9. Pant S, Deshmukh A, Mehta K, Badheka AO, Tuliani T, et al. (2013) Burden of arrhythmias in patients with Takotsubo Cardiomyopathy (apical ballooning syndrome). Int J Cardiol 170: 64-68.

10. Konstantinos M Lampropoulos, Dimitrios Kotsas, Themistoklis A lliopoulos (2012) Apical ballooning syndrome: a case report. 5: 698.

11. Li SL, Wang DX, Wu XM, Li N, Xie YQ (2013) Perioperative acute myocardial infarction increases mortality following noncardiac surgery. J Cardiothorac Vasc Anesth 27: 1277-1281. 